\title{
Establishing Efficient Systems through Electronic Medical Records to Promote Intra-hospital Referrals of Hepatitis Virus Carriers to Hepatology Specialists: A Multicenter Questionnaire-based Survey of 1,281 Healthcare Professionals
}

\author{
Isao Hidaka ${ }^{1}$, Masaru Enomoto ${ }^{2}$, Syuichi Sato $^{3}$, Atsushi Suetsugu ${ }^{4}$, Tomomitsu Matono ${ }^{5}$, \\ Kiyoaki Ito ${ }^{6}$, Koji Ogawa ${ }^{7}$, Jun Inoue ${ }^{8}$, Mika Horino ${ }^{9}$, Yasuteru Kondo ${ }^{10}$, \\ Isao Sakaida $^{1}$ and Masaaki Korenaga ${ }^{11}$
}

\begin{abstract}
:
Objective Persistent hepatitis B virus (HBV) and hepatitis C virus (HCV) infections are major causative factors of hepatic cirrhosis and hepatocellular carcinoma. However, the development of antiviral treatment has enabled their suppression. Therefore, the early detection and treatment of these infections are important. The objective of this study was to assess the level of awareness among healthcare professionals about hepatitis virus infection and electronic medical records alert system.

Methods We surveyed healthcare professionals from 10 institutions with electronic medical records alert systems. All participants attended a lecture about the reactivation risk due to HBV infections, the most recent antiviral treatment for HCV infections, and the electronic medical records alert system. They participated in a questionnaire-based survey about their awareness of these infections, current status of intra-hospital referral, need for intra-hospital referrals before and after the lecture, and reasons for non-referral of patients to specialists.

Results Responses were received from 1,281 healthcare professionals. Physicians and pharmacists had a high level of awareness about HBV and HCV. Among physicians, the level of awareness of those in the surgical field and other fields was significantly lower than that of the professionals in the internal medicine field. The awareness of the need to refer patients to hepatologists increased from $84.7-85.4 \%$ before to $93.0 \%$ after the lecture. The most frequent reasons for not referring patients previously were "I had no knowledge and/or interest" (28.1\% of responses) and "All I did was explain the results orally" $(24.2 \%)$.

Conclusion More widespread education of healthcare personnel is important to increase the number of individuals receiving appropriate treatment from specialist physicians.
\end{abstract}

Key words: awareness survey, non-specialist physicians, promotion of treatment, viral hepatitis

(Intern Med 60: 337-343, 2021)

(DOI: 10.2169/internalmedicine.4748-20)

\footnotetext{
${ }^{1}$ Department of Gastroenterology and Hepatology, Yamaguchi University Hospital, Japan, ${ }^{2}$ Department of Hepatology, Osaka City University Hospital, Japan, ${ }^{3}$ Department of Gastroenterology and Hepatology, Shimane University Hospital, Japan, ${ }^{4}$ Department of Gastroenterology/Internal Medicine, Gifu University Hospital, Japan, ${ }^{5}$ Department of Multidisciplinary Internal Medicine, Tottori University Hospital, Japan, ${ }^{6}$ Department of Gastroenterology, Aichi Medical University, Japan, ${ }^{7}$ Department of Gastroenterology and Hepatology, Hokkaido University Hospital, Japan, ${ }^{8}$ Division of Gastroenterology, Tohoku University Hospital, Japan, ${ }^{9}$ Kochi Medical School Hospital, Japan, ${ }^{10}$ Department of Hepatology, Sendai Kousei Hospital, Japan and ${ }^{11}$ Hepatitis Information Center, Research Center for Hepatitis and Immunology, National Center for Global Health and Medicine, Japan
}

Received for publication March 6, 2020; Accepted for publication May 20, 2020

Correspondence to Dr. Masaru Enomoto, enomoto-m@med.osaka-cu.ac.jp 


\section{Introduction}

According to a 2017 report by the World Health Organization (WHO), the number of people infected with hepatitis $\mathrm{B}$ virus (HBV) or hepatitis $\mathrm{C}$ virus (HCV) worldwide was 325 million, resulting in approximately 1.34 million deaths per year due to hepatocellular carcinoma and end-stage liver diseases (1). In Japan, the number of people with persistent HBV infection was estimated to be 1.12-1.27 million in 2011, and the number of people with persistent HCV infection was estimated to be $0.98-1.58$ million (2). Persistent $\mathrm{HBV}$ and HCV infections are major causative factors for hepatic cirrhosis and hepatocellular carcinoma, which are terminal forms of liver disease $(3,4)$, and in Japan, HBV and/ or HCV cause the majority of hepatocellular carcinoma cases (5). However, good progress has been made in recent years with the development of direct-acting antiviral agents, and antiviral treatment has enabled the suppression of liver disease progression and hepatic carcinogenesis (6-8). Therefore, early detection of these viral infections and early treatment of patients are important; the Japanese Ministry of Health, Labor, and Welfare (MHLW) has recommended that everyone undergoes a hepatitis virus test at least once during their life and that people with positive results should be treated by hepatologists (9).

As the national policy in Japan, local governments are primarily responsible for the tests to ascertain the presence or absence of HBV and HCV infections. In addition, for medical safety, hepatitis virus tests are generally carried out as screening before surgery and invasive tests at medical institutions. Nevertheless, not all people who test positive for hepatitis virus are examined by specialist physicians or receive appropriate treatment (10). In this context, various measures are being taken in all regions of Japan to establish a comprehensive policy for hepatitis treatment, centered on the MHLW, so that hepatitis-positive patients can receive appropriate treatment at medical institutions (11). There have been several reports on the use of an electronic medical records alert system to flag the referral of hepatitis-positive patients to non-specialist physicians; this has been reported to be useful for promoting appropriate examination and treatment of persons with positive results in preoperative and other in-hospital hepatitis virus tests (12-14). Not all patients with positive hepatitis test results are referred to hepatologists solely based on electronic medical records alerts, and it is considered that widespread knowledge about the electronic medical records alert system is important for increasing the intra-hospital referral rate associated with this system.

The present study was, therefore, conducted. At medical institutions where an electronic medical records alert system has been introduced, explanations were given about the alert system through medical safety seminars and so on, and lectures about viral hepatitis were given. In addition to this, a questionnaire-based survey was performed to ascertain whether the knowledge of non-specialist physicians about viral hepatitis and their level of awareness of the need for intra-hospital referral of patients to specialist physicians increased by these measures. The questionnaire also collected information on why those physicians had not previously referred patients to specialist physicians and the factors that constituted barriers to such referrals.

\section{Materials and Methods}

\section{Electronic medical records alert system}

An automatic electronic medical records alert system (HOPE EGMAIN-GX; Fujitsu, Tokyo, Japan) was introduced at Yamaguchi University Hospital in March 2015 (Fig. 1). With this system, if patients are found during an examination, such as preoperative tests, to be positive for hepatitis B surface antigen and/or HCV antibody by nonspecialist physicians (i.e., non-hepatologists), alerts are generated on each patient's screen in the electronic medical records in a fully automated manner to facilitate referral to hepatologists. The alert is cleared by the release of a Results Explanation Form and is therefore displayed until this Form is prepared. Similar systems have been introduced in 10 hospitals, which were the institutions that participated in this study.

\section{Lectures about medical safety}

At each institution where an electronic medical records alert system has been introduced, approximately 30-minute long lectures with shared slides were given to healthcare professionals from various specialties, about the management of $\mathrm{HBV}$, the most recent antiviral treatments for $\mathrm{HCV}$ in medical safety classes, and other relevant information. Explanations were also given about the need for a widespread understanding of the electronic medical records alert system for positive hepatitis virus test results, methods for responding to alerts, and intra-hospital referral for patients who have not undergone regular tests.

\section{Questionnaire-based survey for healthcare profes- sionals}

Lectures were given, and an associated questionnairebased survey was carried out. The survey participants' professions and years of experience were ascertained under anonymized conditions. Using a questionnaire form, before a lecture about the most recent information about HBV/ $\mathrm{HCV}$, the survey participants were asked about their awareness of intra-hospital referral; after the lecture, they were asked about their awareness about the need for intra-hospital referral. This survey was carried out between October 2016 and September 2018. We analyzed the awareness about the topic in each question item among individuals in different specialties such as physicians, pharmacists, nurses, clinical laboratory and radiation technicians, and other professionals, including medical social workers and medical office work- 


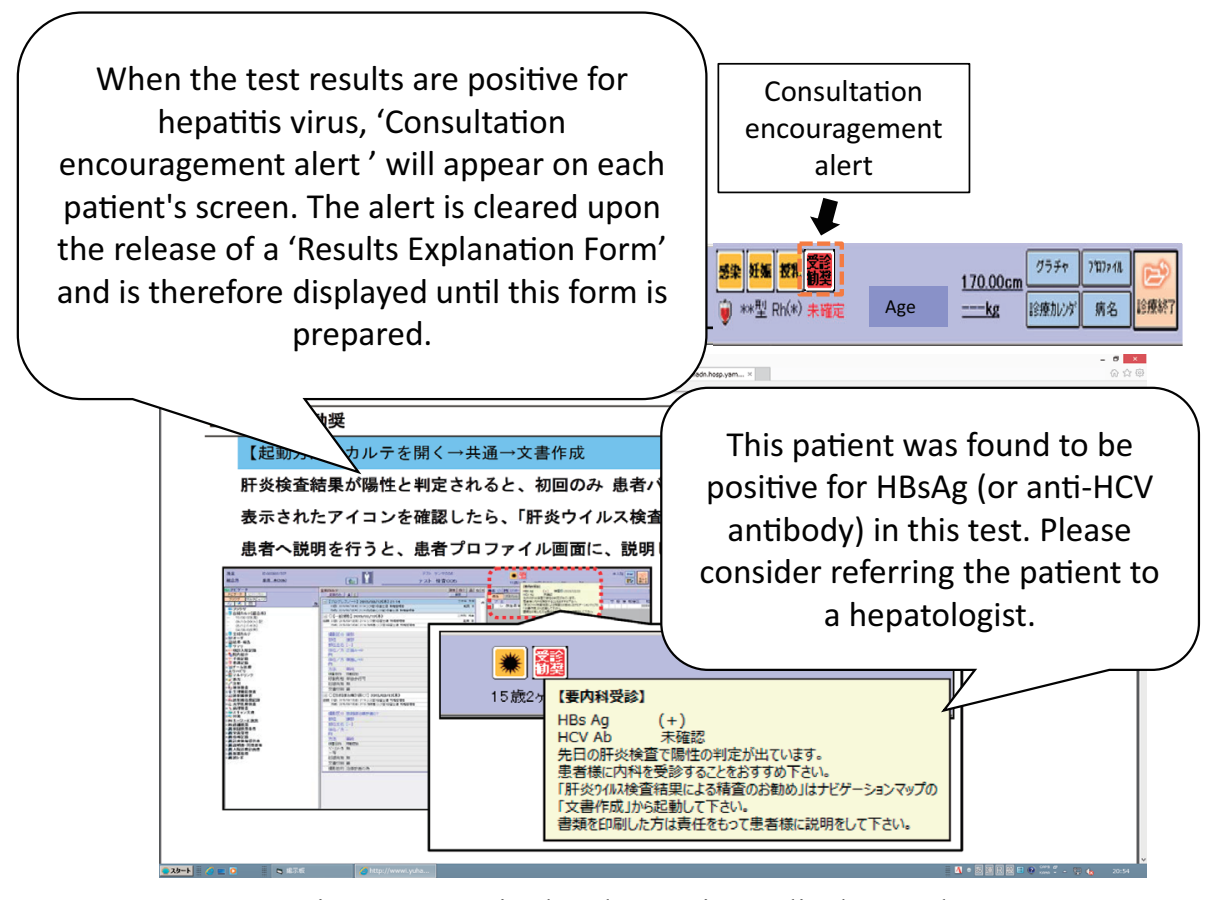

Patient's screen in the electronic medical records

Figure 1. Image of the electronic medical records alert system screen. Patient's screen in the electronic medical records for promoting intra hospital referral of patients positive for hepatitis virus infection at Yamaguchi University Hospital. HBsAg: Hepatitis B surface antigen, HCV: Hepatitis C virus

ers. Furthermore, we compared the awareness about the topics among various physicians in different specialties, such as internal medicine and surgery.

\section{Statistical analysis}

Statistical analysis was performed using JMP Pro software version 13.0.0 (SAS Institute Japan, Tokyo, Japan). The chisquared test was used for between-group comparisons, whereas the Wilcoxon signed-rank test was used to compare the answers before and after the lecture. A p value $<0.05$ was considerered to be significant.

\section{Ethics review}

This study was conducted in accordance with the Declaration of Helsinki. After obtaining approval for the questionnaire-based survey from the Osaka City University Hospital's Institutional Review Board in October 2016, the Institutional Review Board of each participating institution approved it before the survey was carried out.

\section{Results}

The electronic medical records alert system was introduced. The questionnaire-based survey was carried out at the following 10 hospitals: Yamaguchi University Hospital, Osaka City University Hospital, Shimane University Hospital, Gifu University Hospital, Tottori University Hospital, Aichi Medical University Hospital, Hokkaido University Hospital, Tohoku University Hospital, Kochi Medical School
Hospital, and Sendai Kousei Hospital. Training courses, including medical safety seminars, were provided to healthcare professionals in these institutions, and questionnairebased surveys were carried out. The number of professionals who returned the questionnaires was 1,281 , consisting of 458 physicians, 112 pharmacists, 501 nurses, 78 clinical laboratory and radiation technicians, and 132 other professionals.

Awareness about the hepatitis virus in each profession is shown in Fig. 2. The stratification of different awareness levels about the management of HBV was as follows: physicians; "I knew about it completely": 5.1\%; "I largely knew about it": 36.9\%; "I knew a little about it": 55.3\%; and "I did not know anything about it": $4.7 \%$; and pharmacists: "I knew about it completely": $4.5 \%$; "I largely knew about it": 44.6\%; "I knew a little about it": 49.1\%; and "I did not know anything about it": $1.8 \%$. The results for nurses, clinical laboratory technicians, radiology technicians, and other professionals are shown in Fig. 2a.

The stratification of different level of awareness about the most recent direct-acting antiviral agents for treating $\mathrm{HCV}$ was as follows: physicians: "I knew about it completely": 2.9\%; "I largely knew about it": 34.2\%; "I knew a little about it": $56.2 \%$; and "I did not know anything about it": 6.7\%; and pharmacists: "I knew about it completely": $1.8 \%$; "I largely knew about it": 44.1\%; "I knew a little about it": $52.3 \%$; and "I did not know anything about it": $1.8 \%$. The results for nurses, clinical laboratory and radiology technicians, and other professionals are shown in 
(a)

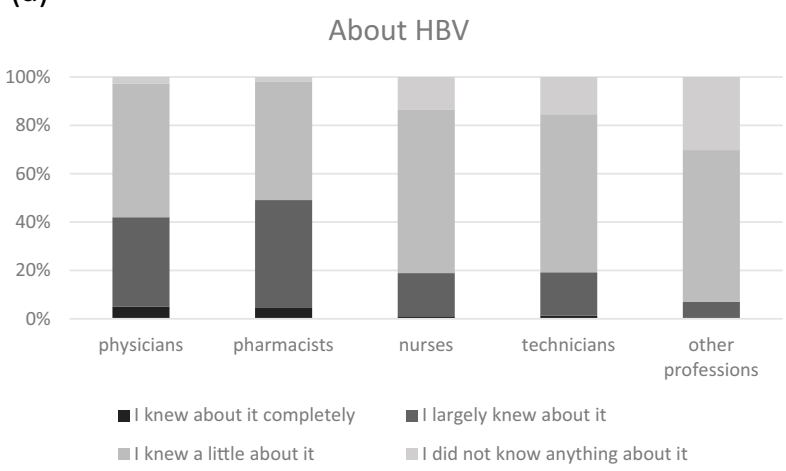

(b)

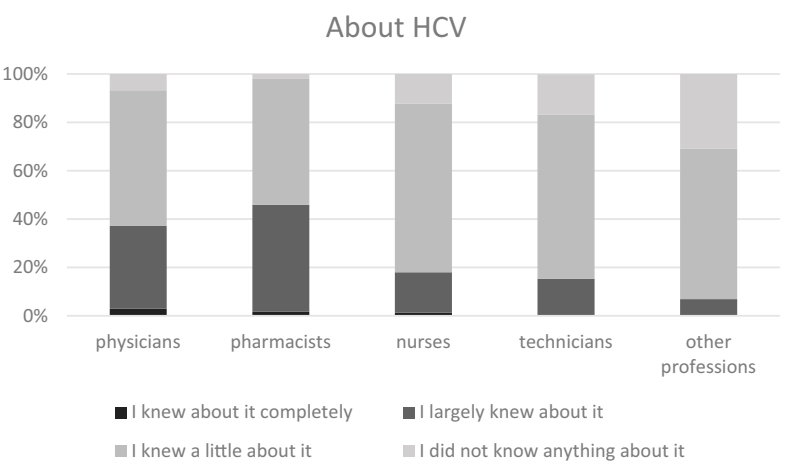

Figure 2. Results of the questionnaire-based survey of the awareness about hepatitis virus, with healthcare professionals classified by profession. Results concerning the awareness about (a) $\mathrm{HBV}$ and (b) HCV. HBV: Hepatitis B virus, HCV: Hepatitis C virus

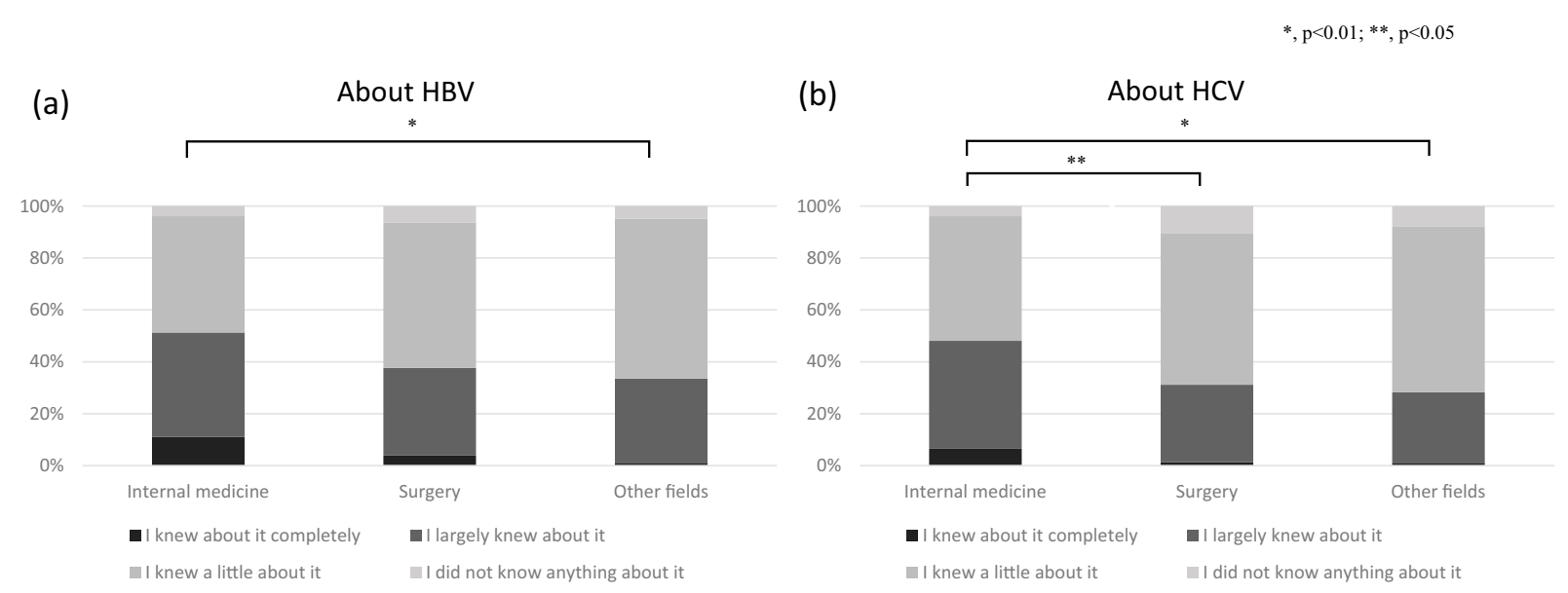

Figure 3. Results of the questionnaire-based survey of the awareness about hepatitis virus, with physicians classified by medical specialty. Results concerning the awareness about (a) HBV and (b) HCV. A Chi-squared test was used for statistical analysis. HBV: Hepatitis B virus, HCV: Hepatitis C virus

Fig. 2b. For both HBV and HCV, physicians and pharmacists had a higher level of awareness than other professionals, and pharmacists, in particular, had a higher level of awareness than non-specialist physicians.

In addition, the level of awareness was compared among physicians of different specialties (Fig. 3). The stratification of physicians in different fields with different awareness levels about the management of HBV was as follows: internal medicine such as cardiovascular medicine and endocrinology: "I knew about it completely": 11.0\%; "I largely knew about it": 40.3\%; "I knew a little about it": 44.8\%; and "I did not know anything about it": $3.9 \%$; surgery such as orthopedic or gastrointestinal surgery: "I knew about it completely": 3.9\%; "I largely knew about it": 33.8\%; "I knew a little about it": $55.8 \%$; and "I did not know anything about it": $6.5 \%$; other fields such as dermatology, ophthalmology, and otorhinolaryngology: "I knew about it completely": 1.1\%; "I largely knew about it": 32.4\%; "I knew a little about it": $61.5 \%$; and "I did not know anything about it": $5.0 \%$. The awareness level among physicians in the surgical fields was lower than that of those in the internal medicine field. The awareness level among physicians in the fields other than surgery and internal medicine was significantly lower $(\mathrm{p}<0.01)$ than that of those in the internal medicine field (Fig. 3a).

The proportions of physicians in different fields with different levels of awareness about direct-acting antiviral agents for treating $\mathrm{HCV}$ were as follows: internal medicine: "I knew about it completely": 6.5\%; "I largely knew about it": 41.6\%; "I knew a little about it": 48.1\%; and "I did not know anything about it": $3.9 \%$; surgery: "I knew about it completely": 1.3\%; "I largely knew about it": 29.9\%; "I knew a little about it": $58.4 \%$; and "I did not know anything about it": 10.4\%; and other fields: "I knew about it completely": 1.1\%; "I largely knew about it": $27.2 \%$; "I knew a little about it": 63.9\%; and "I did not know anything about it": $7.8 \%$. The awareness level of physicians in the surgical fields $(\mathrm{p}<0.05)$ and fields other than surgery and internal medicine $(\mathrm{p}<0.01)$ was significantly lower than that of those in the internal medicine field (Fig. 3b). 
(a) HBV positive patients

Before the lecture

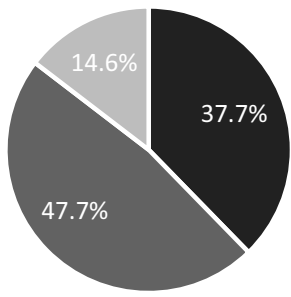

- I have referred all such patients to date

- I have referred such patients when possible

- I have not referred most of such patients

(b) HCV positive patients

\section{Before the lecture}

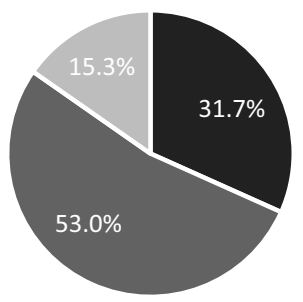

- I have referred all such patients to date

- I have referred such patients when possible

- I have not referred most such patients
After the lecture

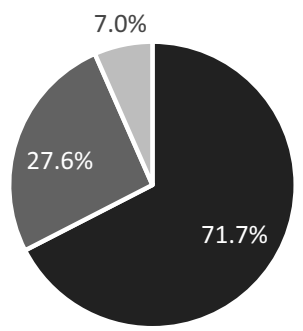

- I intend to refer all such patients in future

- I intend to refer such patients when possible

- I do not intend to refer such patients from now on

\section{After the lecture}

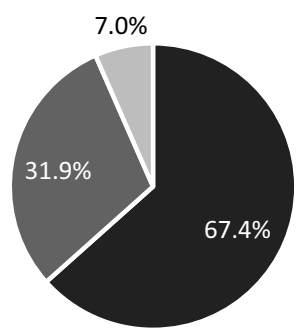

- I intend to refer all such patients in future

- I intend to refer such patients when possible

- I do not intend to refer such patients from now on

Figure 4. Status of referral of hepatitis patients to specialist physicians by non-specialist physicians, and changes in the level of awareness after the lecture. Results (a) about $\mathrm{HBV}$ positive patients and (b) HCV positive patients. A Wilcoxon signed-ranks test was used for statistical analysis. HBV: Hepatitis B virus, HCV: Hepatitis C virus

The referral status of patients positive for hepatitis virus to hepatologists is shown in Fig. 4. Concerning HBVpositive patients, the frequencies of the responses before the lecture were as follows: "I have referred all such patients to date": $37.7 \%$; and "I have referred such patients when possible": $47.7 \%$. After the lecture, these had increased as follows: "I intend to refer all such patients in the future": 71.7\%; and "I intend to refer such patients when possible": $27.6 \%$. Thus, the awareness among physicians about the need to refer patients to specialists had significantly increased to $93.0 \%(p<0.001$, Fig. 4a). Concerning HCVpositive patients, the frequencies of the responses before the lecture were as follows: "I have referred all such patients to date": $31.7 \%$; and "I have referred such patients when possible": $53.0 \%$. After the lecture, these had increased as follows: "I intend to refer all such patients in the future": 67.4\%; and "I intend to refer such patients when possible": $31.9 \%$. Thus, the awareness among physicians about the need to refer patients to specialists had significantly in- creased to $93.0 \%$ ( $<<0.001$, Fig. 4b).

The survey also examined the reasons for not previously referring patients to specialists among physicians. The questionnaire permitted multiple answers, and 432 answers were received. The stratification of physicians giving different responses was as follows: "specialist physicians were already involved": 28.8\%; "I had no knowledge and/or interest": $28.1 \%$; "all I did was explain the results orally": $24.2 \%$; "the patient did not want a referral": 16.4\%; "I have no hepatitis patients": $12.2 \%$; "I have no time to write referral letters": $9.9 \%$; "the primary disease was serious": $8.7 \%$; and "others": $13.9 \%$ (Fig. 5). These survey results show that numerous physicians did not refer patients due to the lack of knowledge and only gave explanations orally; thus, they were unable to explain the results thoroughly or refer patients to specialists. 


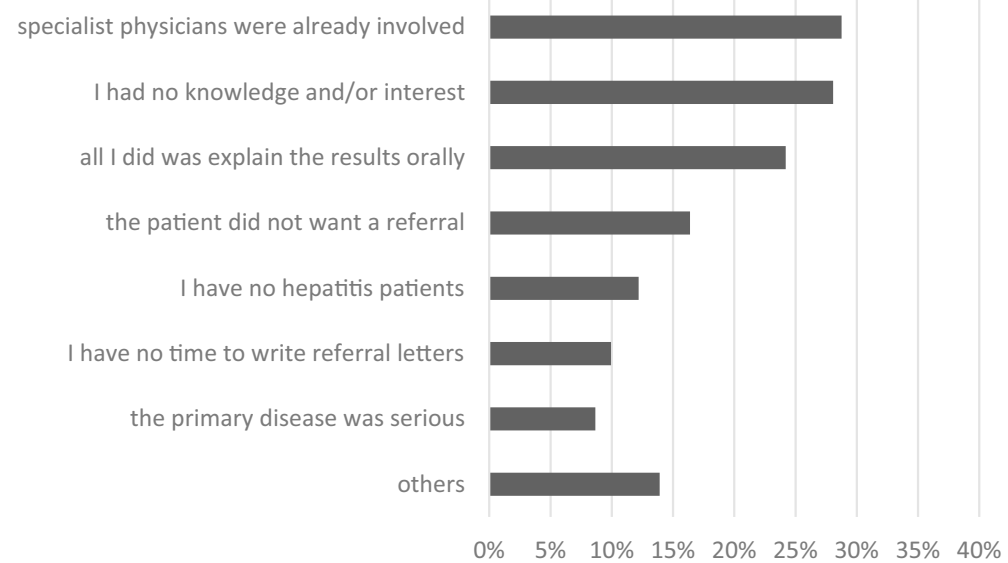

Figure 5. Reasons why non-specialist physicians have not referred hepatitis patients to specialist physicians to date.

\section{Discussion}

Due to the introduction of direct-acting antiviral agents for treating hepatitis $\mathrm{C}$, a high rate of virus elimination has currently become feasible (4), and the number of people with persistent $\mathrm{HCV}$ infection in Japan had decreased to 850,000 as of 2015 (15). Kabiri et al. have reported that if the new test guidelines are followed and the new anti-HCV treatment method is performed reliably, it is expected that by 2036 , the HCV infection rate in the USA will be reduced to approximately 1 in 1,500 , making it a rare disease (16). The WHO has declared a campaign to eradicate viral hepatitis by 2030 (1). In addition, hepatic carcinogenesis is known to be suppressed by virus elimination (17). Similarly, in the case of hepatitis $\mathrm{B}$, disease control can be achieved by oral administration of nucleic acid analogs $(6,7)$. Therefore, it is important to refer patients positive for hepatitis virus to appropriate specialist physicians so that treatment can be initiated at an early stage and/or appropriate tests can be conducted regularly.

In Japan, national policies have been established to ensure that hepatitis patients receive appropriate treatment (11), with one of these policies being the promotion of hospitalized treatment. Furthermore, the recommendation of treatment using an electronic medical records alert system has been reported to be useful (12-14). As an attempt similar to that in Japan, Konerman et al. developed a "best-practice recommendation" system using electronic health records and reported this to be a useful tool for promoting treatment (18). This study is the first report in our country that surveyed several institutions. The finding of the present questionnaire-based survey was that the proportion of nonspecialist physicians who are aware of the most recent information about HBV/HCV was only $37.1 \%$ to $42.0 \%$ (Fig. 2). The level of awareness about the most recent information among physicians in the surgical field and fields other than surgery and internal medicine, who are considered to have numerous opportunities to conduct hepatitis virus tests as screening before surgery or invasive tests, was particularly low. In addition, $28.1 \%$ of the physicians stated "I had no knowledge and/or interest" as the reason why they had not referred patients to specialists. There have been reports of similar results in questionnaire-based surveys of dialysis specialists (19), and it is considered that increasing awareness will be connected to the appropriate medical institutions. In this study, lectures were explicitly given to all healthcare workers, including the physicians in the medical safety class, where attendance was indispensable to all institutions. Therefore, we considered that our employed publicity measures for physicians not interested in an intrahospital referral system was effective. In this study, we only investigated the current referral trend before the lecture and the consequent intention (intention to refer patients to specialists) after the lecture; we could not examine the behavioral modification concerning referrals after the lecture. We intend to investigate in the future, whether, and how these lectures improve the rate of the introduction to specialists.

Because nurses have more opportunities to interact with patients, we expect synergy between the lecture and encouragement to advise patients to consult non-specialists by the nurses. Furthermore, this questionnaire-based survey showed that pharmacists had a higher level of awareness than nonspecialist physicians about the management of HBV and antiviral treatment for hepatitis C. Because pharmacists are often concerned with patient compliance to antiviral therapy and $\mathrm{HBV}$ reactivation measures, we infer that a pharmacist has abundant knowledge on viral hepatitis. To establish an organization such that, together with dealing with HBV reactivation, recommendations for treatment by specialist physicians can be made not only by non-specialist physicians, but also by pharmacists. Moreover, further measures have to be taken so that knowledge about this area can be spread to all healthcare professionals, and that hepatitis patients can undergo treatment at appropriate medical institutions at an early stage (20). In addition, this survey showed that other professionals had the lowest level of awareness about HBV and $\mathrm{HCV}$ because most of them were medical office work- 
ers who lacked technical knowledge about the disease. We believe that the lecture would be useful for medical office workers when an explanation of the specific medical expenses subsidy system for patients with hepatitis is provided to them.

We performed an attitude survey about knowledge on viral hepatitis and the need for intra-hospital referral to a specialist, which also involved the use of a medical records alert system to encourage the in-hospital referral of patients with hepatitis to a specialist. We believe that non-specialist physicians are not adequately informed, and the resultant effect of that is the restrictive use of the medical records alert system. Therefore, lectures are essential to provide further insight to non-specialists. Furthermore, the promotion of referrals can be expected from a team approach in medical care, resultant from such lectures delivered to several categories of healthcare workers. We intend to examine the intervention by the team approach in medical care, as a further aid to physicians who are not acquainted with the medical records alert system.

\section{Conclusion}

The outcome of this questionnaire-based survey suggests that the limited awareness about the most recent information on hepatitis viruses on the part of non-specialist physicians is a factor inhibiting the intra-hospital referral of patients to appropriate specialists. An electronic medical records alert system is useful for the intra-hospital referral of HBV and $\mathrm{HCV}$ carriers to hepatologists; however, to be effectively used, it is important to disseminate information about the system and provide education to increase awareness about viral hepatitis.

The authors state that they have no Conflict of Interest (COI).

\section{Financial Support}

This research was supported financially by the Ministry of Health, Labour and Welfare, Japan (grant number: H29-kanseiippan-004).

\section{Acknowledgement}

We would like to thank all the healthcare professionals from the 10 medical institutions that participated in our questionnairebased survey.

\section{References}

1. World Health Organization. Hepatitis [Internet]. [cited 2020 Feb 9]. Available from: https://www.who.int/hepatitis/about/en/.

2. Tanaka J, Akita T, Ko K, Miura Y, Satake M; Epidemiological Research Group on Viral Hepatitis and its Long-term Course Ministry of Health, Labour and Welfare of Japan. Countermeasures against viral hepatitis B and C in Japan: an epidemiological point of view. Hepatol Res 49: 990-1002, 2019.

3. European Association for the Study of the Liver. EASL 2017 clinical practice guidelines on the management of hepatitis B virus infection. J Hepatol 67: 370-398, 2017.

4. European Association for the Study of the Liver. EASL recommendations on treatment of hepatitis C 2018. J Hepatol 69: 461$511,2018$.

5. Tateishi R, Uchino K, Fujiwara N, et al. A nationwide survey on non-B, non-C hepatocellular carcinoma in Japan: 2011-2015 update. J Gastroenterol 54: 367-376, 2019.

6. Liaw YF, Sung JJY, Chow WC, et al. Lamivudine for patients with chronic hepatitis B and advanced liver disease. N Engl J Med 351: 1521-1531, 2004.

7. Hosaka T, Suzuki F, Kobayashi M, et al. Long-term entecavir treatment reduces hepatocellular carcinoma incidence in patients with hepatitis B virus infection. Hepatology 58: 98-107, 2013.

8. Waziry R, Hajarizadeh B, Grebely J, et al. Hepatocellular carcinoma risk following direct-acting antiviral HCV therapy: a systematic review, meta-analyses, and meta-regression. J Hepatol 67: 1204-1212, 2017.

9. Mitchell AE, Colvin HM, Beasley RP. Institute of Medicine recommendations for the prevention and control of hepatitis B and C. Hepatology 51: 729-733, 2010.

10. Furukawa N, Kawaguchi $\mathrm{Y}$, Oeda $\mathrm{S}$, et al. Current management practices for HBs antigen or anti-HCV antibody positive individuals in non-hepatology departments at a university hospital. Kanzo 54: 307-316, 2013 (in Japanese with English abstract).

11. Oza N, Isoda H, Ono T, Kanto T. Current activities and future directions of comprehensive hepatitis control measures in Japan: the supportive role of the Hepatitis Information Center in building a solid foundation. Hepatol Res 47: 487-496, 2017.

12. Shimomura Y, Ikeda F, Nouso K, et al. Introduction of management system of positive test results for HBV and HCV infection in electronic medical chart. Kanzo 56: 137-143, 2015 (in Japanese with English abstract).

13. Uchida-Kobayashi S, Enomoto M, Fujii $\mathrm{H}$, et al. Promotion of intra-hospital referral of hepatitis B and C virus carriers to hepatology specialists by electronic medical record-based alert system: a case study at a university hospital. Kanzo 57: 7-16, 2016 (in Japanese with English abstract).

14. Fujii H, Yamaguchi S, Kurai O, et al. Putting "sticky notes" on the electronic medical record to promote intra-hospital referral of hepatitis $\mathrm{B}$ and hepatitis $\mathrm{C}$ virus-positive patients to hepatology specialists: an expoloratory study. BMC Infect Dis 16: 410, 2016.

15. The Polaris Observatory HCV Collaborators. Global prevalence and genotype distribution of hepatitis $\mathrm{C}$ virus infection in 2015: a modelling study. Lancet Gastroenterol Hepatol 2: 161-176, 2017.

16. Kabiri M, Jazwinski AB, Roberts MS, Schaefer AJ, Chhatwal J. The changing burden of hepatitis $\mathrm{C}$ virus infection in the United States: model-based predictions. Ann Intern Med 161: 170-180, 2014.

17. Kanwal F, Kramer J, Asch SM, Chayanupatkul M, Cao Y, El-Serag HB. Risk of hepatocellular cancer in $\mathrm{HCV}$ patients treated with direct-acting antiviral agents. Gastroenterology 153: 996-1005.e1, 2017.

18. Konerman MA, Thomson M, Gray K, et al. Impact of an electronic health record alert in primary care on increasing hepatitis $\mathrm{c}$ screening and curative treatment for baby boomers. Hepatology 66: 1805-1813, 2017.

19. Okubo T, Atsukawa M, Tsubota A, et al. Epidemiological survey of patients with hemodialysis complicated by hepatitis C in Japan. Ther Apher Dial 23: 44-48, 2019.

20. Inoue T, Urano S, Inoue I, Korenaga M, Tanaka Y. Recommendation of medical consultation and/or treatment by pharmacists to patients with chronic hepatitis $\mathrm{C}$ who visited health insurance pharmacies. Kanzo 58: 639-642, 2017 (in Japanese with English abstract).

The Internal Medicine is an Open Access journal distributed under the Creative Commons Attribution-NonCommercial-NoDerivatives 4.0 International License. To view the details of this license, please visit (https://creativecommons.org/licenses/ by-nc-nd/4.0/).

(C) 2021 The Japanese Society of Internal Medicine

Intern Med 60: 337-343, 2021 